\title{
Exploring the effect of exercise training on testicular function
}

\section{Bárbara Matos $^{1,2}$, John Howl ${ }^{3}$, Rita Ferreira ${ }^{1}$, Margarida Fardilha ${ }^{2}$}

${ }^{1}$ QOPNA, Departamento de Química, Universidade de Aveiro, Campus Universitário de Santiago, 3810-193 Aveiro, Portugal

2 Signal Transduction Laboratory, iBiMED, Department of Medical Sciences, University of Aveiro, 3810-193 Aveiro, Portugal

${ }^{3}$ Molecular Pharmacology Group, Research Institute in Healthcare Science, University of Wolverhampton, Wolverhampton WV1 1LY, UK

\section{Corresponding author}

Margarida Fardilha: mfardilha@ua.pt

\section{ACKNOWLEDGMENTS}

We are thankful to the Portuguese Foundation for Science and Technology (FCT), European Union, QREN, FEDER and COMPETE for funding the iBiMED (UID/BIM/04501/2013 and POCI-01-0145-FEDER-007628), QOPNA (UID/QUI/00062/2013) and CIAFEL (UID/DTP/00617/2013) research units, and the research project RUNawayPCa (POCI-01-0145-FEDER-006958 and PTDC/DTPDES/6077/2014).

\section{ABSTRACT}

Purpose: The impact of exercise training on testicular function is relatively ill-defined. To gain new insights into this important topic, published data, deriving from both humans and animal studies, were critically analyzed.

Results and Conclusions: The effects of exercise on the hypothalamus-pituitary-gonadal axis, influenced by the type, intensity and duration of the exercise program, can be evaluated in terms of total and free testosterone and/or luteinizing hormone (LH) and follicle-stimulating hormone (FSH) serum levels and sperm parameters. High intensity exercise promotes a common decrease in these parameters and so negatively impacts upon testicular function. However, published data for moderate-intensity exercise training are inconsistent. Conversely, there is consistent evidence to support the benefits 
of exercise training to prevent and/or counteract the impairment of testis function caused by aging, obesity and doxorubicin treatment. This positive effect is likely the consequence of decreased oxidative stress and inflammatory status. In the future it will be important to clarify the molecular mechanisms which explain these reported discrepancies and to establish guidelines for an active lifestyle to promote healthy testicular function.

Keywords: physical activity, hypothalamus-pituitary-gonadal axis, testosterone, aging, obesity.

\section{LIST OF ABREVIATIONS}

$3 \beta$-HSD - 3 $\beta$-hydroxysteroid dehydrogenase

17ß-HSD - 17ß-hydroxysteroid dehydrogenase

$\mathrm{BMI}$ - body mass index

CAT - catalase

DNA - deoxyribonucleic acid

DOX - doxorubicin

$\mathrm{FSH}$ - follicle-stimulating hormone

G6PDH - glucose-6-phosphate dehydrogenase

GPx - glutathione peroxidase

GST - glutathione S-transferase

HCR - high capacity runners

HPG - hypothalamus-pituitary-gonadal

HSP70 - heat shock protein 70

IL-1 $\beta$ - interleukin-1 $\beta$

IL-10 - interleukin-10

JAK2 - Janus kinase 2

LCR - Low intrinsic capacity runners

LDH - lactate dehydrogenase

LEP - leptin

$\mathrm{LH}$ - luteinizing hormone

MDA - malondialdehyde 
METs - metabolic equivalents

NADPH - nicotinamide adenine dinucleotide phosphate

NF-kB - nuclear factor kappa B

Nrf2 - nuclear factor erythroid 2p45-related factor 2

ODF-1 - outer dense fiber protein 1

$\mathrm{PO}_{2 m}$ - microvascular oxygen partial pressure

SAMP8 - senescence-accelerated prone mouse model

SHBG - sex hormone binding globulin

SOD -superoxide dismutase

StAR - steroidogenic acute regulatory protein

STAT3 - signal transducer and activator of transcription 3

TGF- $\alpha$ - transforming growth factor alpha

TNF- $\alpha$ - tumor necrosis factor alpha

TUNEL - transferase nick-end labelling

$\mathrm{VO}_{2 \max }$ - maximal oxygen uptake

\section{INTRODUCTION}

There is consensus amongst the scientific and clinical communities that exercise improves an individual's health and quality of life. Increasingly, exercise training is being promoted by physicians as a primary life-style prevention for several diseases, particularly those of the cardiovascular system (Xu et al. 2010; Boyle et al. 2012; Wu et al. 2013; Aune et al. 2015; Vasankari et al. 2017). However, the recommendation of exercise training as a preventive or therapeutic strategy for testicular dysfunction is more problematic since data relating the impact of exercise training on testicular function are controversial.

Impairment of testicular function has increased in industrialized countries with a consequential decrease in fertility rates, coupled with additional negative clinical outcomes that compromise life quality. Indeed, additional physiological functions may be influenced by chronic changes in the levels of testosterone, a steroid hormone with a panoply of essential systemic roles that include stabilization of blood sugar and maintenance of cardiovascular function (Köhn 2006). Thus, decreased levels of testosterone may increase the propensity of developing serious disease states which 
include type 2 diabetes and cardiovascular pathologies (Traish et al. 2009; Corona et al. 2011).

Lifestyle and behavioral causes of testicular dysfunction include modifiable factors such as obesity that can be altered or avoided (Sharpe 1974). Indeed, men with a body mass index (BMI) higher than 25 have an average $25 \%$ reduction in sperm number and motility compared with those exhibiting a normal BMI (Kort et al. 2006). In this case, exercise training has been recommended to reduce and control body mass (Swift et al. 2014); however, it might not be an appropriate intervention to manage testis dysfunction in these patients. Moreover, aging is also recognized as a cause of testicular dysfunction since it is associated with decreased free testosterone levels (Orwoll et al. 2006).

Herein, we rigorously review the current knowledge on the cross-talk between exercise training and testis function with particular reference to the systemic and molecular alterations promoted by exercise training of different types and intensities. Most of these studies focused upon aerobic exercise training, running and swimming programmes of moderate- and high-intensity and employ animal models to evaluate the molecular alterations in testis. In human studies, the molecular markers of testicular dysfunction were generally evaluated in blood-derived fluids. Subsequently, we discuss the effect of exercise training in both healthy individuals and those with impaired testicular function caused by distinct pathophysiological conditions. These findings support the definition of guidelines for the prescription of exercise training in the case of individuals with or at risk of testicular dysfunction.

\section{MODERATE-INTENSITY EXERCISE TRAINING}

Several population based-studies reported a positive association between moderate-intensity aerobic exercise training and testis function parameters such as sperm concentration and hormonal serum levels. Indeed, increased serum levels of total and free testosterone, sperm count, motility and morphology were observed in trained subjects (Grandys et al. 2009; Vaamonde et al. 2012; Gaskins et al. 2015; Priskorn et al. 2016). The HPG axis may be responsible for the observed alterations, since LH and FSH were also increased, suggesting an increased stimulation of Leydig and Sertoli cells' 
functions (Grandys et al. 2009; Vaamonde et al. 2012; Gaskins et al. 2015; Priskorn et al. 2016). Alterations in sex hormone binding globulin (SHBG) serum levels were also observed after exercise training; however, there is no consensus about the trend of such alterations since both a decrease and an increase in its levels were reported. This globulin binds to testosterone, reducing its bioavailability (Grandys et al. 2009; Priskorn et al. 2016). Resistance training, despite being less intensively studied, seems also to have beneficial influence upon testicular function. This exercise regime was associated with increased levels of total and free testosterone serum levels (Ahtiainen et al. 2003), similar to those verified for aerobic training. Endurance activities have been the most studied, particularly those of moderate intensity. A maximal oxygen uptake $\left(\mathrm{VO}_{2 \max }\right)$ of 50\% (range 40-60\%) (Grandys et al. 2009; Vaamonde et al. 2012), or 3-6 metabolic equivalents (METs), a measure of energy consumption, is usually considered in the definition of moderate-intensity exercise programs (Gaskins et al. 2015; Priskorn et al. 2016).

Contrary findings were described in other studies, with no impact or negative effects of moderate-intensity exercise training on testicular function (Safarinejad et al. 2009; Dominguez et al. 2011; Wise et al. 2011; Torma et al. 2014). In a longitudinal study, lower serum levels of free and total testosterone, LH and FSH were observed in moderateintensity trained men, suggesting decreased testis function. Higher levels of SHBG were also observed in trained subjects, accordingly with reduced levels of free testosterone serum levels (Safarinejad et al. 2009). Wise et al. (Wise et al. 2011) demonstrated a lack of association between exercise training of diverse types, including running and cycling (aerobic) and weightlifting (resistance), at different hours per week, and semen parameters. One exception was observed in a population of men who cycled as a primary form of exercise. These subjects presented a decreased sperm count and motility if they cycled for five or more hours per week when compared with a cohort who did not exercised regularly. So, the effect of exercise training seems to depend not only on the type but also on the duration of the activity. Furthermore, long-term cycling seems to be associated with a negative outcome though the precise underlying mechanisms responsible are unknown. Testicular mechanical trauma, the result of compression against the bicycle saddle, and increased scrotal temperature may both induce an inflammatory response with negative outcomes in testis function. Several saddle designs 
that reduce the pressure in the testis have been proposed to minimize the influence of this confounding factor when analyzing the effect of this type of exercise training on testicular function (Rodano et al. 2002; Lowe et al. 2004).

To better comprehend the molecular basis of the physiological alterations in testis promoted by exercise training in men, animal model-based studies have been designed and performed (figure 1). Moderate-intensity treadmill running was reported to induce a weakened oxygen supply to rat testis tissue, since a decreased microvascular oxygen partial pressure $\left(\mathrm{PO}_{2 \mathrm{~m}}\right)$ value was observed in the testis of trained rats. These lower levels of $\mathrm{PO}_{2 m}$ may cause vascular dysfunction, which contributes to the generation of an anoxic environment. The resulting perturbations in intracellular milieu in turn impact upon the health and function of the testis (Dominguez et al. 2011). An impaired spermatogenesis was also suggested, since decreased levels of outer dense fiber protein 1 (ODF-1) were also observed following treadmill training. ODF-1 is required for the correct morphology of sperm, being essential for a rigid head-tail junction (Yang et al. 2012; Torma et al. 2014).

\section{HIGH-INTENSITY EXERCISE TRAINING}

There is a general awareness that high-intensity exercise training is harmful to testicular function (figure 1). A high intensity exercise program is defined by a $\mathrm{VO}_{2 \max }$ higher than $60 \%$ or more than 6 METs. One randomized controlled longitudinal study involved 362 habitual aerobic men athletes, aged 20-40 years, submitted to 60 weeks of a running program ( 5 sessions/week, $120 \mathrm{~min} / \mathrm{session}$ at speed adjusted to $\mathrm{VO}_{2 \max }$ of $80 \%)$. In this study, lower serum levels of total and free testosterone, higher levels of SHBG, and decreased semen parameters like sperm count, motility and morphology were observed (Safarinejad et al. 2009). These hormonal and physiological alterations are suggestive of impaired testicular function, which may be associated with an effect in HPG axis, since decreased serum levels of LH and FSH were also observed. Lower levels of $\mathrm{LH}$ and $\mathrm{FSH}$, together with decreased testosterone levels and sperm parameters indicate that either there is an impairment of GnRH secretion by the hypothalamus or GnRH did not stimulate the anterior pituitary to secrete LH and FSH.

Studies with animal models indicate a negative impact of high-intensity exercise training on hormonal and seminal parameters (Manna et al. 2003, 2004; Jana et al. 2008; 
Chigrinskiy and Conway 2011). The decrease in testosterone serum levels was corroborated by a decreased testis activity of some steroidogenic enzymes, including $3 \beta$ -

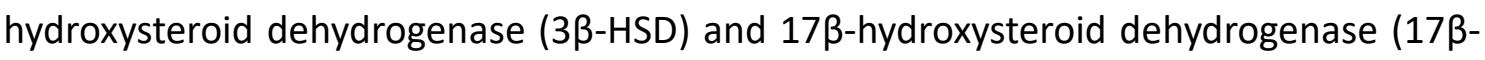
HSD), responsible for several steps of testosterone production. Yet the involvement of the HPG axis in these alterations is controversial, with some studies reporting no significant alterations in FSH and decreased LH serum levels (Manna et al. 2003, 2004), and others reporting increased LH serum levels (Chigrinskiy and Conway 2011). Despite considering the same type of exercise the duration and frequency of exercise training programs differed amongst studies and these factors may, at least in part, explain the contradictory results.

Several hypotheses have been proposed to explain the harmful effects of highintensity exercise training upon testicular function. One such hypothesis is predicated upon the detrimental influences of exercise-induced oxidative stress. Several authors have described a decreased activity of enzymatic antioxidant defenses, including superoxide dismutase (SOD), catalase (CAT), glutathione peroxidase (GPx) and glutathione S-transferase (GST), plus a reduced content of the non-enzymatic antioxidant defenses glutathione (GSH) and ascorbic acid in rat testis after swimming programs lasting 4 weeks ( 3 hours/day, 5 days/week), 5 weeks (daily, until exhausting) or 10 weeks (4 hours/day, 6 days/week) (Manna et al. 2003, 2004; Jana et al. 2008; Chigrinskiy and Conway 2011). In agreement with data showing a decrease in antioxidant defenses, increased levels of some markers of oxidative damage were also observed, especially markers of lipid peroxidation (malondialdehyde (MDA) and conjugated dienes), suggesting that lipid peroxidation may contribute to the testicular dysfunction caused by intensive exercise training (Manna et al. 2003, 2004; Jana et al. 2008; Chigrinskiy and Conway 2011). Ribose deficiency was also suggested as a possible mechanism that contributes to testis dysfunction in exercised subjects. A decrease in ribose was confirmed by reduced levels of rat testis glucose-6-phosphate dehydrogenase (G6PDH), the enzyme that catalyzes the first step of the pentose phosphate pathway. Reduced levels of this enzyme may be associated with increased oxidative stress since decreased G6PDH inhibits nicotinamide adenine dinucleotide phosphate (NADPH) generation to reduce the activity of glutathione reductase, the enzyme that regenerates GSH from GSSG. Accordingly, oral administration of D-ribose to Wistar rats, caused a 
restoration of testis MDA, GSH and ascorbic acid, and increased testosterone and LH serum levels (Chigrinskiy and Conway 2011). Finally, the lower levels of heat shock protein 70 (HSP70) observed in rat spermatogenic cells after high-intensity exercise training may be responsible, at least in part, for impaired spermatogenesis (Koeva et al. 2005). Decreased levels of HSP70 were associated with maturation arrest in the germinative cell populations, which in some cases causes low sperm count and may be associated with male infertility (Feng et al. 2001).

\section{EFFECT OF EXERCISE TRAINING IN TESTICULAR DYSFUNCTION ASSOCIATED WITH:}

\section{AGING}

Longitudinal studies strongly associate aging with testicular dysfunction, the likely consequence of age-related increases in oxidative stress and inflammation (Wang et al. 2005; Luo et al. 2006). Several randomized control trials have associated both aerobic and resistance exercise of moderate and high intensity with improvement in sperm parameters in infertile men, indicating improvement of testicular function in these patients(Maleki and Tartibian 2017; Maleki et al. 2017). Similarly, in older men, exercise was associated with a transient increase in testosterone serum levels, counteracting agerelated testicular dysfunction (Zmuda et al. 1996). Studies using a senescenceaccelerated prone mouse model (SAMP8) submitted, from two months of age, to a moderate-intensity swimming exercise program lasting 6 months, reported a protection and/or reversion of age-associated impairments of testicular function (figure 2) (Chigurupati et al. 2008; Zhao et al. 2013; Joseph et al. 2014). Exercised mice presented higher levels of nuclear factor erythroid 2p45-related factor 2 (Nrf-2) in testis, which leads to an increased expression of antioxidant enzymes, confirmed by the increased mRNA levels of SOD and GPx. The activities of these two enzymes, like CAT, were also increased in the testis of exercised mice. In agreement with these results, a decreased oxidative damage in the testis of exercised animals, determined by lower levels of protein carbonyls, 8-isoprostanes, MDA and 8-hydroxy-20-deoxyguanosine, was observed (Zhao et al. 2013). In addition to the modulation of oxidative stress, a reduction in testis inflammation after exercise training was also observed based on a decreased 
levels of nuclear factor kappa B (NF-kB), a transcription factor which induces the expression of pro-inflammatory cytokines, chemokines and additional inflammatory mediators. Accordingly, reduced levels of pro-inflammatory mediators, interleukin-1 $\beta$ (IL-1 $\beta)$ and tumor necrosis factor alpha (TNF- $\alpha$ ), and increased levels of testis antiinflammatory mediators, interleukin-10 (IL-10) and transforming growth factor alpha (TGF- $\alpha$ ), confirmed the anti-inflammatory effect of lifelong exercise training in the testis (Zhao et al. 2013). The exercise training-related reduction of oxidative stress and inflammation in testis improved testicular function. Indeed, lifelong exercised rats presented increased levels of serum and testis testosterone. These findings were corroborated by the observed higher levels of- steroidogenic enzymes, steroidogenic acute regulatory protein (StAR) and Cyp11a, which are responsible for the first steps of steroidogenesis. In addition, an increased number of Leydig cells and spermatogonia in seminiferous tubules were reported, observations which suggest increased testosterone and sperm production respectively (Chigurupati et al. 2008; Zhao et al. 2013). Exercised older rats showed attenuated age-related testicular atrophy and reduced levels of testis DNA damage, measured by the levels of phosphorylated histone H2AX (Joseph et al. 2014). The benefits of exercise training in counteracting age-related testis dysfunction are more pronounced when performed early in life (Zhao et al. 2013).

\section{OBESITY}

Obesity is also correlated with testicular dysfunction, highlighted by decreased serum levels of free testosterone, a result of decreased gonadotropins, coupled with impaired spermatogenesis (Stokes et al. 2015). In obese men, weight loss as a result of exercise training improves semen quality and the production of reproductive hormones (Hakonsen et al. 2011). Obese male mice with impaired spermatogenesis evidenced increased levels of serum leptin (LEP), a finding indicative of leptin resistance. Indeed, reduced testis expression of leptin receptors and of two precursors of leptin signal transduction: Janus kinase 2 (JAK2) and signal transducer and activator of transcription 3 (STAT3), were observed in obese mice, resulting in reduced activation of the LEP/JAK2/STAT3 signal transduction pathway (Yi et al. 2017). To the best of our knowledge, only one study has evaluated the effect of exercise training in obesityinduced testis dysfunction using a mice model submitted to eight weeks of swimming 
training. This exercise training program restored the LEP-JAK-STAT signaling pathway in testis. (figure 2) The same study revealed an increased expression of steroidogenic factor-1, which controls the transcription of some steroidogenic enzymes, including StAR and Cyp11a, also elevated in trained obese mice. Accordingly, an increased level of serum total testosterone, an increased sperm count and an improvement in sperm motility, were also observed (Yi et al. 2017).

\section{DOXORUBICIN TREATMENT}

Testicular dysfunction is a reported side-effect of some drugs. One example is doxorubicin (DOX), a chemotherapy agent used to treat several types of cancer. DOX treatment impairs testis function, confirmed by both a reduced number and diameter of testicular seminiferous tubules and the depletion of spermatids in DOX-treated rats. To date, there are two main mechanisms proposed to be responsible for DOX-induced testis dysfunction: lipid peroxidation and cellular apoptosis. Indeed, increased MDA levels, decreased SOD, GPx and GSH levels, and increased terminal deoxynucleotidyl transferase nick-end labelling (TUNEL)-positive cells suggesting increased cell apoptosis through detection of deoxyribonucleic acid (DNA) fragmentation, were observed in testis tissue of DOX-treated rats (Yeh et al. 2009; Magalhães et al. 2017). A recent study, using rats sub-chronically treated with DOX ( $2 \mathrm{mg} / \mathrm{kg}$ per week, during 7 week), 5 weeks after initiating a treadmill exercise program, showed no positive effect of exercise training to prevent the reduced sperm motility induced by DOX or to alleviate oxidative damage in the testis. Nevertheless, beneficial influence of exercise training to inhibit the oxidative damage of proteins involved in metabolism and the stress response against DOX was suggested (figure 2) (Magalhães et al. 2017). To the best of our knowledge, there are no studies performed in human subjects treated with chemotherapeutic agents.

\section{LOW INTRINSIC CAPACITY RUNNERS RATS}

The low intrinsic capacity runners (LCR) rat exhibits impaired testicular function and some authors have used this model to evaluate the effect of exercise training in the modulation of testicular dysfunction (Torma et al. 2014). This animal model is characterized by lower sirtuin-1 levels in the testis, associated with attenuation of spermatogenesis (Coussens et al. 2008). Paradoxically to its known deacetylase function, 
sirtuin-1 was associated with the acetylation of histones. The morphological alterations necessary to generate spermatozoa from haploid spermatid cells include the histone to protamine transition, a process that is initiated with post-translational modifications of histones, including acetylation. Thus, reduced sirtuin-1 levels reduce this acetylation process and, consequently, impair spermatogenesis (Coussens et al. 2008).

After twelve weeks of treadmill running at $60 \% \mathrm{VO}_{2 \max }$ (moderate-intensity exercise training), LCR rats presented an enhancement in markers of testis and sperm function, but sirtuin-1 remained unchanged (Torma et al. 2014). Decreased levels of testis reactive oxygen species (ROS) and increased ODF-1 were observed, suggesting an improvement in spermatogenesis (figure 2). An increase in lactate dehydrogenase (LDH) C levels were also observed in testis after exercise training. This testis-specific isoform of LDH is essential for germ cells energy metabolism since lactate is a key energetic substrate in these cells. Consequently, LDHC plays a key role in sperm function (Odet et al. 2008). Curiously, these apparent beneficial alterations caused by exercise training were not verified in high capacity runners (HCR), who did not suffer from testicular dysfunction. Hence, in comparison with LCR rats, a contrary effect was observed for ODF-1, which decreased with exercise training in HCR rats (Torma et al. 2014).

\section{CONCLUSIONS AND FUTURE PERSPECTIVES}

The andrological aspects of exercise training taking into consideration the possible risks and benefits for reproductive and sexual health, should be considered when playing sport. Exercise training might positively or negatively influence andrological health depending upon the type, intensity and duration of the training program, and on individual health status. This is particularly relevant when a lifestyle-related disease influencing reproductive and sexual functions (e.g. obesity, metabolic syndrome, diabetes and cardiovascular diseases) is prevalent. Curiously, in the presence of a condition that impairs testicular function, like obesity or DOX treatment, data from the literature is consensual showing that exercise training prevents and/or counteracts the negative effect of the underlying condition on testis function. Decreased oxidative stress and, possibly, inflammation following an exercise training program seems to markedly contribute to this beneficial effect of exercise.

In conclusion, we recommend that future studies should seek to further clarify the 
many effects of exercise training on testicular function and elucidate their underlying molecular mechanisms. We envision that such findings will provide guidelines to enable physicians to recommend a more appropriate active lifestyle to prevent, or even to treat testicular dysfunction associated with aging, obesity and drug treatments.

\section{REFERENCES}

Ahtiainen JP, Pakarinen A, Alen M, et al (2003) Muscle hypertrophy, hormonal adaptations and strength development during strength training in strength-trained and untrained men. Eur J Appl Physiol 89:555-563. doi: 10.1007/s00421-003-08333

Aune D, Norat T, Leitzmann M, et al (2015) Physical activity and the risk of type 2 diabetes: A systematic review and dose-response meta-analysis. Eur J Epidemiol 30:529-542. doi: 10.1007/s10654-015-0056-Z

Boyle T, Keegel T, Bull F, et al (2012) Physical activity and risks of proximal and distal colon cancers: A systematic review and meta-analysis. J Natl Cancer Inst 104:15481561. doi: $10.1093 /$ jnci/djs354

Chigrinskiy EA, Conway VD (2011) Protective effect of D-ribose against inhibition of rats testes function at excessive exercise. J Stress Phhysiology Biochem 7:242-249

Chigurupati S, Son TG, Hyun DH, et al (2008) Lifelong running reduces oxidative stress and degenerative changes in the testes of mice. J Endocrinol 199:333-341. doi: 10.1677/JOE-08-0306

Corona G, Rastrelli G, Monami M, et al (2011) Hypogonadism as a risk factor for cardiovascular mortality in men: A meta-analytic study. Eur J Endocrinol 165:687701. doi: 10.1530/EJE-11-0447

Coussens M, Maresh JG, Yanagimachi R, et al (2008) Sirt1 deficiency attenuates spermatogenesis and germ cell function. PLoS One 3:1-8. doi: 10.1371/journal.pone.0001571

Dominguez JM, Davis RT, McCullough DJ, et al (2011) Aging and exercise training reduce testes microvascular $\mathrm{PO} 2$ and alter vasoconstrictor responsiveness in testicular arterioles. Am J Physiol 301:R801-10. doi: 10.1152/ajpregu.00203.2011

Feng HL, Sandlow JI, Sparks AET (2001) Decreased expression of the heat shock protein 
hsp70-2 is associated with the pathogenesis of male infertility. Fertil Steril 76:11361139. doi: 10.1016/S0015-0282(01)02892-8

Gaskins AJ, Mendiola J, Afeiche M, et al (2015) Physical activity and television watching in relation to semen quality in young men. Br J Sports Med 49:265-270. doi: 10.1136/bjsports-2012-091644

Grandys M, Majerczak J, Duda K, et al (2009) Endurance training of moderate intensity increases testosterone concentration in young, healthy men. Int J Sports Med 30:489-495. doi: 10.1055/s-0029-1202340

Hakonsen L, Thulstrup A, Aggerholm A, et al (2011) Does weight loss improve semen quality and reproductive hormones results from a cohort of severely obese men. Reprod Health 8:1-8. doi: 10.1186/1742-4755-8-24

Jana K, Samanta PK, Manna I, et al (2008) Protective effect of sodium selenite and zinc sulfate on intensive swimming-induced testicular gamatogenic and steroidogenic disorders in mature male rats. Appl Physiol Nutr Metab 33:903-14. doi: $10.1139 / \mathrm{H} 08-065$

Joseph AM, Nguyen LMD, Welter AE, et al (2014) Mitochondrial adaptations evoked with exercise are associated with a reduction in age-induced testicular atrophy in Fischer-344 rats. Biogerontology 15:517-534. doi: 10.1007/s10522-014-9526-Z

Koeva Y, Delchev S, Georgieva K, Atanassova P (2005) Heat Shock protein-70 expression in testis following endurance training of rats. Proc Balk Sci Conf Biol Plovdiv 288-294.

Köhn FM (2006) Testosterone and body functions. Aging Male 9:183-188. doi: $10.1080 / 13685530601060396$

Kort HI, Massey JB, Elsner CW, et al (2006) Impact of body mass index values on sperm quantity and quality. J Androl 27:450-452. doi: 10.2164/jandrol.05124

Lowe BD, Schrader SM, Breitenstein MJ (2004) Effect of bicycle saddle designs on the pressure to perineum of the bicyclist. Med Sci Sports Exerc 36:1055-1062. doi: 10.1249/01.MSS.0000128248.40501.73

Luo L, Chen H, Trush MA, et al (2006) Aging and the brown Norway rat leydig cell antioxidant defense system. J Androl 27:240-7. doi: 10.2164/jandrol.05075

Magalhães J, Ascensão A, Padrão AI, et al (2017) Can exercise training counteract doxorubicin-induced oxidative damage of testis proteome? Toxicol Lett 280:57-69. doi: $10.1016 /$ j.toxlet.2017.08.010

Maleki BH, Tartibian B (2017) High-Intensity Exercise Training for Improving 
Reproductive Function in Infertile Patients: A Randomized Controlled Trial. J Obstet Gynaecol Canada 39:545-558. doi: 10.1016/j.jogc.2017.03.097

Maleki BH, Tartibian B, Hajizadeh Maleki B, et al (2017) Moderate aerobic exercise training for improving reproductive function in infertile patients: A randomized controlled trial. Cytokine 92:55-67. doi: 10.1016/j.cyto.2017.01.007

Manna I, Jana K, Samanta PK (2003) Effect of intensive exercise-induced testicular gametogenic and steroidogenic disorders in mature male Wistar strain rats: A correlative approach to oxidative stress. Acta Physiol Scand 178:33-40. doi: 10.1046/j.1365-201X.2003.01095.x

Manna I, Jana K, Samanta PK (2004) Effect of different intensities of swimming exercise on testicular oxidative stress and reproductive dysfunction in mature male albino Wistar rats. Indian J Exp Biol 42:816-822

Odet F, Duan C, Willis WD, et al (2008) Expression of the Gene for Mouse Lactate Dehydrogenase C (Ldhc) Is Required for Male Fertility1. Biol Reprod 79:26-34. doi: 10.1095/biolreprod.108.068353

Orwoll E, Lambert LC, Marshall LM, et al (2006) Testosterone and estradiol among older men. J Clin Endocrinol Metab 91:1336-1344. doi: 10.1210/jc.2005-1830

Priskorn L, Jensen TK, Bang AK, et al (2016) Is Sedentary Lifestyle Associated with Testicular Function? A Cross-Sectional Study of 1,210 Men. Am J Epidemiol 184:284-294. doi: 10.1093/aje/kwv338

Rodano R, Squadrone R, Sacchi M, Marzegan A (2002) Saddle Pressure Distribution in Cycling: Comparison Among Saddles of Different Design and Materials. ISBS Conf Proc Arch 1:606-609

Safarinejad MR, Azma K, Kolahi AA (2009) The Effects of intensive, long-term treadmill running on reproductive hormones, hypothalamus-pituitary-testis axis, and semen quality: A randomized controlled study. J Endocrinol 200:259-271. doi: 10.1677/JOE-08-0477

Sharpe R (1974) Environmental Causes of Testicular Dysfunction. In: Male hypogonadism. 281-304

Stokes VJ, Anderson RA, George JT (2015) How does obesity affect fertility in men and what are the treatment options. Clin Endocrinol (Oxf) 25:633-638. doi: $10.1111 /$ cen. 12591

Swift DL, Johannsen NM, Lavie CJ, et al (2014) The Role of Exercise and Physical Activity in Weight Loss and Maintenance. Prog Cardiovasc Dis 56:441-447. doi: 
10.1016/j.pcad.2013.09.012

Torma F, Koltai E, Nagy E, et al (2014) Exercise increases markers of spermatogenesis in rats selectively bred for low running capacity. PLoS One 9:1-11. doi: 10.1371/journal.pone.0114075

Traish AM, Saad F, Guay A (2009) The dark side of testosterone deficiency: II. type 2 diabetes and insulin resistance. J Androl 30:23-32. doi: 10.2164/jandrol.108.005751

Vaamonde D, Da Silva-Grigoletto ME, García-Manso JM, et al (2012) Physically active men show better semen parameters and hormone values than sedentary men. Eur J Appl Physiol 112:3267-3273. doi: 10.1007/s00421-011-2304-6

Vasankari V, Husu P, Vähä-Ypyä H, et al (2017) Association of objectively measured sedentary behaviour and physical activity with cardiovascular disease risk. Eur J Prev Cardiol 24:1311-1318. doi: 10.1177/2047487317711048

Wang X, Shen CL, Dyson MT, et al (2005) Cyclooxygenase-2 regulation of the agerelated decline in testosterone biosynthesis. Endocrinology 146:4202-4208. doi: 10.1210/en.2005-0298

Wise LA, Cramer DW, Hornstein MD, et al (2011) Physical activity and semen quality among men attending an infertility clinic. Fertil Steril 95:1025-1030. doi: 10.1016/j.fertnstert.2010.11.006

Wu Y, Zhang D, Kang S (2013) Physical activity and risk of breast cancer: A metaanalysis of prospective studies. Breast Cancer Res Treat 137:869-882. doi: $10.1007 / \mathrm{s} 10549-012-2396-7$

Xu Q, Park Y, Huang X, et al (2010) Physical activities and future risk of Parkinson disease. Neurology 75:341-348. doi: 10.1212/WNL.0b013e3181ea1597

Yang K, Meinhardt a., Zhang B, et al (2012) The Small Heat Shock Protein ODF1/HSPB10 Is Essential for Tight Linkage of Sperm Head to Tail and Male Fertility in Mice. Mol Cell Biol 32:216-225. doi: 10.1128/MCB.06158-11

Yeh Y, Liu T, Wang L, et al (2009) A standardized extract of Ginkgo biloba suppresses doxorubicin-induced oxidative stress and p53-mediated mitochondrial apoptosis in rat testes. Br J Pharmacol 156:48-61. doi: 10.1111/j.1476-5381.2008.00042.x

Yi X, Gao H, Chen D, et al (2017) Effects of obesity and exercise on testicular leptin signal transduction and testosterone biosynthesis in male mice. Am J Physiol - Regul Integr Comp Physiol 312:R501-R510. doi: 10.1152/ajpregu.00405.2016

Zhao X, Bian Y, Sun Y, et al (2013) Effects of moderate exercise over different phases on age-related physiological dysfunction in testes of SAMP8 mice. Exp Gerontol 
48:869-880. doi: 10.1016/j.exger.2013.05.063

Zmuda JM, Thompson PD, Winters SJ (1996) Exercise increases serum testosterone and sex hormone- binding globulin levels in older men. Metabolism 45:935-939. doi: 10.1016/S0026-0495(96)90258-9 


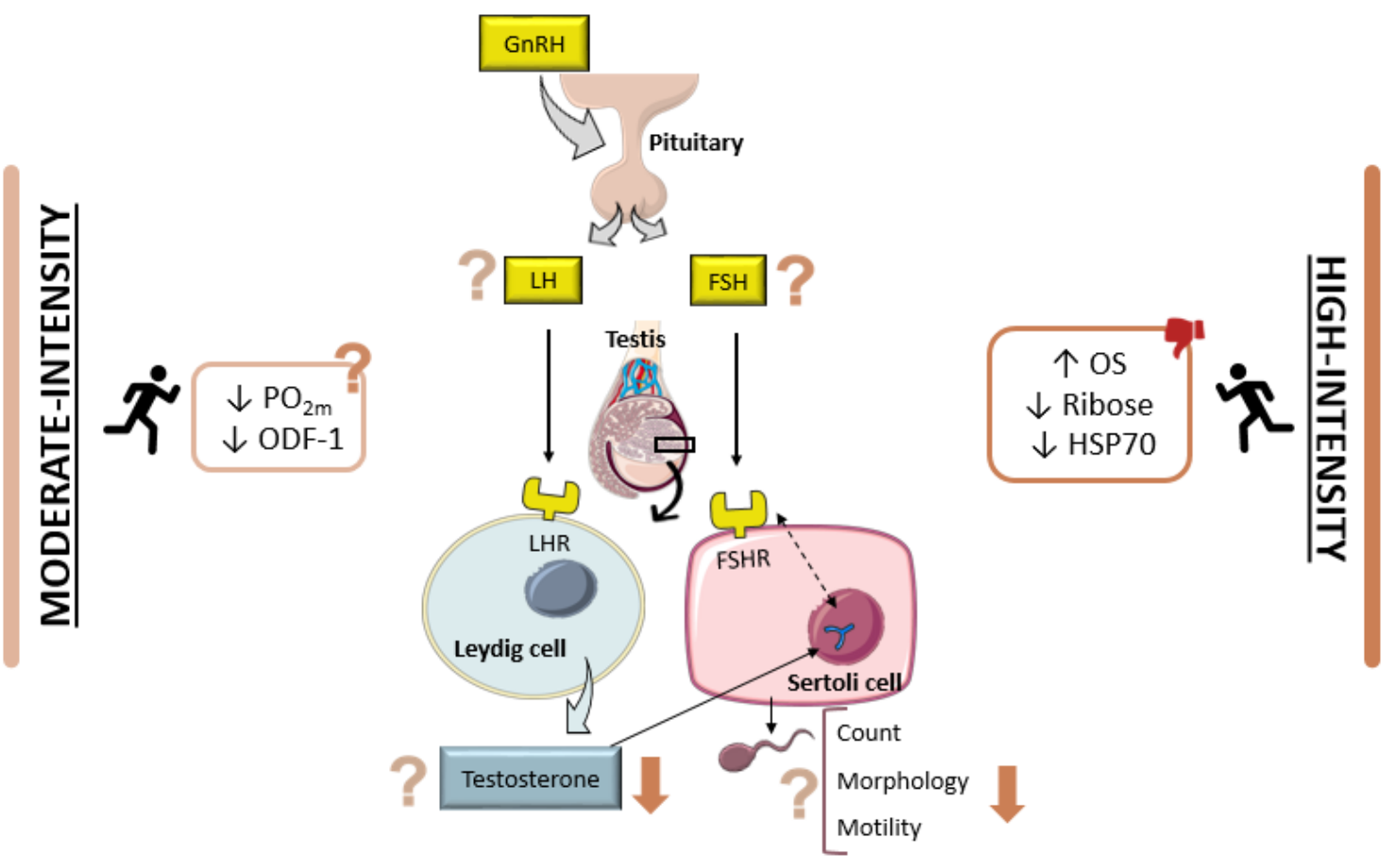

Figure 1: Overview of the impact of moderate- and high-intensity exercise training in testicular function. The effect of moderate-intensity exercise training in testicular function is not consensual among scientific community but a decrease in $\mathrm{PO}_{2 \mathrm{~m}}$ and ODF-1 after moderate-intensity exercise training is proposed. High-intensity exercise training negatively affect testis function, decreasing testosterone levels and sperm count and impairing sperm morphology and motility. Increased levels of OS and decreased levels of ribose and HSP70 are proposed as responsible mechanisms.

Abbreviations: GnRH: gonadotropin-releasing hormone; LH: luteinizing hormone; FSH: follicle-stimulating hormone; LHR: LH receptor; FSHR: FSH receptor; $\mathbf{P O}_{2} \mathbf{m}$ : microvascular oxygen partial pressure; ODF-1: outer dense fiber protein 1; OS: oxidative stress; HSP70: heat shock protein 70

Figure was produced using Servier Medical Art. 


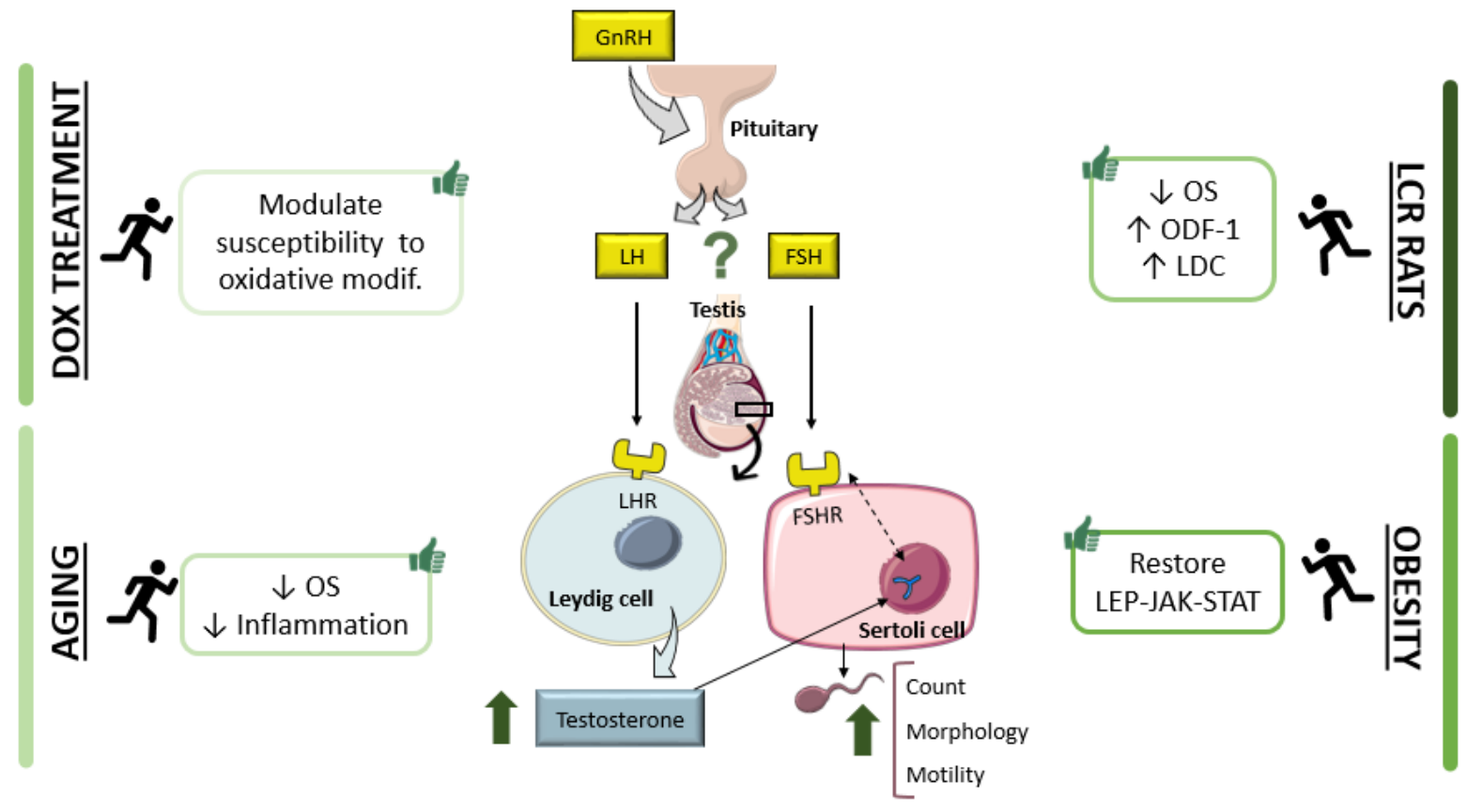

Figure 2: Overview of the impact of exercise training on testicular function in the presence of a condition that negatively affects these functions. DOX treatment negatively affects testicular function and exercise training improves testosterone levels and sperm parameters by modulating the susceptibility to oxidative modifications. With aging, testicular function is affected and exercise training helps to counteract these negative alterations by decreasing oxidative stress and inflammation. LCR rats are characterized by an impaired testicular function and, when subject to exercise training, an improvement in testicular function is observed. Decreased OS and increased ODF-1 and LDC are responsible for the above effect. A restoration of LEP-JAK-STAT signaling pathway after exercise training is observed in obese men, with consequent improvement of testis function parameters.

Abbreviations: GnRH: gonadotropin-releasing hormone; LH: luteinizing hormone; FSH: follicle-stimulating hormone; LHR: LH receptor; FSHR: FSH receptor; ET: exercise training; ODF-1: outer dense fiber protein 1; OS: oxidative stress; LDC: lactate dehydrogenase C; LEP-JAK-STAT: leptin-Janus kinase 2-signal transducer and activator of transcription 3 signaling pathway; DOX: doxorubicin; LCR: low capacity runners.

Figure was produced using Servier Medical Art. 